\title{
A NONLINEAR ERGODIC THEOREM FOR A REVERSIBLE SEMIGROUP OF LIPSCHITZIAN MAPPINGS IN A HILBERT SPACE
}

\author{
HAJIME ISHIHARA AND WATARU TAKAHASHI
}

(Communicated by John B. Conway)

\begin{abstract}
Let $C$ be a nonempty closed convex subset of a Hilbert space, $S$ a right reversible semitopological semigroup, $\mathscr{S}=\left\{T_{t}: t \in S\right\}$ a continuous representation of $S$ as Lipschitzian mappings on a closed convex subset $C$ into $C$, and $F(\mathscr{S})$ the set of common fixed points of mappings $T_{t}, t \in S$. Then we deal with the existence of a nonexpansive retraction $P$ of $C$ onto $F(\mathscr{S})$ such that $P T_{t}=T_{t} P=P$ for each $t \in S$ and $P x$ is contained in the closure of the convex hull of $\left\{T_{t} x: t \in S\right\}$ for each $x \in C$.
\end{abstract}

1. Introduction. Let $C$ be a closed convex subset of a real Hilbert space $H$ and $T$ be a mapping of $C$ into itself. $T$ is said to be a Lipschitzian mapping if for each $n \geq 1$ there exists a positive real number $k_{n}$ such that

$$
\left\|T^{n} x-T^{n} y\right\| \leq k_{n}\|x-y\|
$$

for all $x, y \in C$. A Lipschitzian mapping is said to be nonexpansive if $k_{n}=1$ for all $n \geq 1$ and asymptotically nonexpansive if $\lim _{n} k_{n}=1$, respectively. We denote by $F(T)$ the set of fixed points of $T$. The first nonlinear ergodic theorem for nonexpansive mappings was established in 1975 by Baillon [1]: Let $C$ be a closed convex subset of a Hilbert space and $T$ be a nonexpansive mapping of $C$ into itself. If the set $F(T)$ is nonempty, then for each $x \in C$, the Cesàro means

$$
S_{n}(x)=\frac{1}{n} \sum_{k=0}^{n-1} T^{k} x
$$

converge weakly to some $y \in F(T)$. In this case, putting $y=P x$ for each $x \in C$, $P$ is a nonexpansive retraction of $C$ onto $F(T)$ such that $P T=T P=P$ and $P x \in$ $\overline{\mathrm{co}}\left\{T^{n} x: n=0,1,2, \ldots\right\}$ for each $x \in C$, where $\overline{\mathrm{co}} A$ is the closure of the convex hull of $A$. In [11 and 12], Takahashi proved the existence of such a retraction-"ergodic retraction" - for an amenable and a right reversible semigroup of nonexpansive mappings in a Hilbert space. On the other hand, Hirano and Takahashi [4] proved the Cesàro means for asymptotically nonexpansive mappings converge weakly to a fixed point.

In this paper, we deal with the existence of "ergodic retraction" for a right reversible semigroup of Lipschitzian mappings; that is, we prove a nonlinear ergodic theorem for such a semigroup in a Hilbert space. This theorem is a generalization

Received by the editors June 20, 1987.

1980 Mathematics Subject Classification (1985 Revision). Primary 47A35; Secondary 47H09.

Key words and phrases. Ergodic theorem, reversible semigroup, asymptotically nonexpansive mapping, fixed point. 
of the result $[\mathbf{4}, \mathbf{1 2}]$. Furthermore, we prove a weak convergence theorem which is similar to that of $[\mathbf{7}, \mathbf{9}]$.

2. Nonlinear ergodic theorem. Let $S$ be a semitopological semigroup, i.e., $S$ is a semigroup with a Hausdorff topology such that for each $a \in S$ the mappings $s \rightarrow a \cdot s$ and $s \rightarrow s \cdot a$ from $S$ to $S$ are continuous. Let $C$ be a nonempty closed convex subset of a Hilbert space $H$. Then a family $\mathscr{S}=\left\{T_{t}: t \in S\right\}$ of mappings from $C$ into itself is said to be a Lipschitzian semigroup on $C$ if $\mathscr{S}$ satisfies the following:

(1) $T_{t s}(x)=T_{t} T_{s}(x)$ for $t, s \in S$ and $x \in C$;

(2) the mapping $(s, x) \rightarrow T_{s}(x)$ from $S \times C$ into $C$ is continuous when $S \times C$ has the product topology;

(3) for each $s \in S$, there exists $k_{s}>0$ such that $\left\|T_{s}(x)-T_{s}(y)\right\| \leq k_{s}\|x-y\|$ for $x, y \in C$.

A semitopological semigroup $S$ is right reversible if any two closed left ideals of $S$ have nonvoid intersection. In this case, $(S, \leq)$ is a directed system when the binary relation " $\leq$ " on $S$ is defined by $a \leq b$ if and only if $\{a\} \cup \overline{S a} \supseteq\{b\} \cup \overline{S b}$. Let $F(\mathscr{S})$ denote the set $\left\{x \in C: T_{s} x=x\right.$ for all $\left.s \in S\right\}$ of common fixed points of mappings $T_{s}, s \in S$ in $C$ (fixed point theorems for Lipschitzian semigroups were proved in $[6,3])$. Then we have the following:

THEOREM 1. Let $C$ be a closed convex subset of a real Hilbert space $H$ and let $S$ be a right reversible semitopological semigroup. Let $\mathscr{S}=\left\{T_{t}: t \in S\right\}$ be a Lipschitzian semigroup on $C$ with $\lim \sup _{s} k_{s} \leq 1$. Then $F(\mathscr{S})$ is a closed convex subset of $C$.

ProOF. Closedness of $F(\mathscr{S})$ is obvious. To show convexity it is sufficient to prove that $z=(x+y) / 2 \in F(\mathscr{S})$ for all $x, y \in F(\mathscr{S})$. We have

$$
\left\|T_{t} z-x\right\|^{2}=\left\|T_{t} z-T_{t} x\right\|^{2} \leq k_{t}^{2}\|z-x\|^{2}=\frac{1}{4} k_{t}^{2}\|x-y\|^{2}
$$

and

Thus

$$
\left\|T_{t} z-y\right\|^{2}=\left\|T_{t} z-T_{t} y\right\|^{2} \leq k_{t}^{2}\|z-y\|^{2}=\frac{1}{4} k_{t}^{2}\|x-y\|^{2} .
$$

$$
\left\|T_{t} z-z\right\|^{2}=\frac{1}{2}\left\|T_{t} z-x\right\|^{2}+\frac{1}{2}\left\|T_{t} z-y\right\|^{2}-\frac{1}{4}\|x-y\|^{2} \leq \frac{1}{4}\left(k_{t}^{2}-1\right)\|x-y\|^{2}
$$

and hence $\lim _{t}\left\|T_{t} z-z\right\|=0$. Therefore we obtain

$$
z=\lim _{t} T_{t} z=\lim _{t} T_{s t} z=\lim _{t} T_{s} T_{t} z=T_{s} \lim _{t} T_{t} z=T_{s} z
$$

for all $s \in S$.

Let $\left\{x_{\alpha}: \alpha \in A\right\}$ be a bounded net of a Hilbert space $H$ and let $C$ be a closed convex subset of $H$. Then we define

$$
r(x)=\lim _{\beta} \sup _{\beta \leq \alpha}\left\|x_{\alpha}-x\right\| \quad \text { and } \quad r=\inf \{r(x): x \in C\} .
$$

It is well known that there exists a unique element $a \in C$ with $r(a)=r$, called the asymptotic center of $\left\{x_{\alpha}: \alpha \in A\right\}$ in $C$. A useful lemma is a result proved in [5], which we state here as:

LEMMA 1. $r^{2}+\|a-x\|^{2} \leq r(x)^{2}$ for every $x \in C$.

As a consequence, we have the following. 
LEMMA 2 (LIM [8]). Let $\left\{y_{\beta}\right\}$ be a net of $C$ such that $\lim \sup _{\beta} r\left(y_{\beta}\right) \leq r$. Then $y_{\beta} \rightarrow a$.

PROOF. By Lemma 1, we have $r^{2}+\left\|a-y_{\beta}\right\|^{2} \leq r\left(y_{\beta}\right)^{2}$ and hence

$$
r^{2}+\limsup _{\beta}\left\|a-y_{\beta}\right\|^{2} \leq \underset{\beta}{\limsup } r\left(y_{\beta}\right)^{2} \leq r^{2} .
$$

Therefore we have $\lim \sup _{\beta}\left\|a-y_{\beta}\right\|^{2}=0$ and this implies $y_{\beta} \rightarrow a$.

We also know that if $\left\{x_{\alpha}\right\} \subseteq C$ and if $\left\{x_{\alpha}\right\}$ converges weakly to $y \in C$ then $y=a[2$, Theorem 4.2]. Let $Q$ be the metric projection of $H$ onto $F(\mathscr{S})$. Then, by Phelps [10], $Q$ is nonexpansive. Before proving a nonlinear ergodic theorem, we prove the following crucial lemma.

LEMMA 3. Let $C$ be a closed convex subset of a real Hilbert space $H$ and let $S$ be a right reversible semitopological semigroup. Let $\mathscr{S}=\left\{T_{t}: t \in S\right\}$ be a Lipschitzian semigroup on $C$ with $\lim \sup _{s} k_{s} \leq 1$. Suppose that $F(\mathscr{S}) \neq \varnothing$. Then for each $x \in C,\left\{Q T_{s} x: s \in S\right\}$ converges to the asymptotic center of $\left\{T_{s} x: s \in S\right\}$ in $F(\mathscr{S})$.

ProOF. Let $z$ be the asymptotic center of $\left\{T_{s} x: s \in S\right\}$ in $F(\mathscr{S})$. Then, for all $s, t \in S$ we obtain

$$
\begin{aligned}
r\left(Q T_{s} x\right) & \leq \sup _{a \geq t s}\left\|T_{a} x-Q T_{s} x\right\|=\sup _{a \geq t}\left\|T_{a s} x-Q T_{s} x\right\| \\
& =\sup _{a \geq t}\left\|T_{a} T_{s} x-T_{a} Q T_{s} x\right\| \leq\left(\sup _{a \geq t} k_{a}\right)\left\|T_{s} x-Q T_{s} x\right\| \\
& \leq\left(\sup _{a \geq t} k_{a}\right)\left\|T_{s} x-z\right\|
\end{aligned}
$$

and hence

$$
r\left(Q T_{s} x\right) \leq \limsup _{t} k_{t}\left\|T_{s} x-z\right\| \leq\left\|T_{s} x-z\right\|
$$

for all $s \in S$. Therefore we have $\lim \sup _{s} r\left(Q T_{s} x\right) \leq \lim \sup _{s}\left\|T_{s} x-z\right\| \leq r$. By Lemma 2, we obtain $Q T_{s} x \rightarrow z$.

THEOREM 2. Let $C$ be a closed convex subset of a Hilbert space $H$ and let $S$ be a right reversible semitopological semigroup. Let $\mathscr{S}=\left\{T_{t}: t \in S\right\}$ be a Lipschitzian semigroup on $C$ with $\lim \sup _{s} k_{s} \leq 1$. Suppose that

$$
F(\mathscr{S})=\bigcap\left\{F\left(T_{s}\right): s \in S\right\} \neq \varnothing .
$$

Then the following are equivalent:

(a) $\bigcap_{s \in S} \overline{\operatorname{co}}\left\{T_{t} x: t \geq s\right\} \cap F(\mathscr{S}) \neq \varnothing$ for each $x \in C$.

(b) There is a nonexpansive retraction $P$ of $C$ onto $F(\mathscr{S})$ such that $P T_{t}=$ $T_{t} P=P$ for every $t \in S$ and $P x \in \overline{\mathrm{co}}\left\{T_{t} x: t \in S\right\}$ for every $x \in C$.

ProOF. (b) $\Rightarrow(\mathrm{a})$. Let $x \in C$. Then $P x \in F(\mathscr{S})$. Also

$$
P x \in \bigcap_{s \in S} \overline{\operatorname{co}}\left\{T_{t} x: t \geq s\right\} .
$$

In fact,

$$
P x=P T_{s} x \in \overline{c o}\left\{T_{t} T_{s} x: t \in S\right\} \subset \overline{c o}\left\{T_{t} x: t \geq s\right\}
$$

for every $s \in S$. 
(a) $\Rightarrow(\mathrm{b})$. Let $x \in C$ and $f \in F(\mathscr{S})$. Then for each $s, t \in S$, we have

$$
\begin{aligned}
\operatorname{lim\operatorname {sup}}\left\|T_{a} x-f\right\| & \leq \sup _{a \geq t s}\left\|T_{a} x-f\right\|=\sup _{a \geq t}\left\|T_{a s} x-f\right\| \\
& =\sup _{a \geq t}\left\|T_{a} T_{s} x-T_{a} f\right\| \leq\left(\sup _{a \geq t} k_{a}\right)\left\|T_{s} x-f\right\|
\end{aligned}
$$

and hence

$$
\underset{a}{\limsup }\left\|T_{a} x-f\right\| \leq\left(\limsup _{t} k_{t}\right)\left\|T_{s} x-f\right\| \leq\left\|T_{s} x-f\right\|
$$

for every $s \in S$. So $\lim \sup _{s}\left\|T_{s} x-f\right\| \leq \liminf _{s}\left\|T_{s} x-f\right\|$ and hence the $\lim _{s}\left\|T_{s} x-f\right\|$ exists. Let $Q$ be the metric projection of $H$ onto $F(\mathscr{S})$. Then by Lemma 3, $\left\{Q T_{s} x\right\}$ converges to the asymptotic center $z$ of $\left\{T_{s} x: s \in S\right\}$ in $F(\mathscr{S})$. Let $u \in \bigcap_{s \in S} \overline{\operatorname{co}}\left\{T_{t} x: t \geq s\right\} \cap F(\mathscr{S})$. Then, since

$$
\|z-u\|^{2}=\left\|T_{s} x-u\right\|^{2}-\left\|T_{s} x-z\right\|^{2}-2\left\langle z-u, T_{s} x-z\right\rangle
$$

for every $s \in S$, we have

$$
\begin{aligned}
\|z-u\|^{2}+2 \lim _{s}\left\langle z-u, T_{s} x-z\right\rangle & =\lim _{s}\left\|T_{s} x-u\right\|^{2}-\lim _{s}\left\|T_{s} x-z\right\|^{2} \\
& =\limsup _{s}\left\|T_{s} x-u\right\|^{2}-\underset{s}{\limsup }\left\|T_{s} x-z\right\|^{2} \\
& =r(u)^{2}-r^{2} \geq 0 .
\end{aligned}
$$

Let $\varepsilon>0$. Then we have

$$
2 \lim _{s}\left\langle z-u, T_{s} x-z\right\rangle>-\|z-u\|^{2}-\varepsilon .
$$

Hence there exists $s_{0} \in S$ such that

$$
2\left\langle z-u, T_{s} x-z\right\rangle>-\|z-u\|^{2}-\varepsilon
$$

for every $s \geq s_{0}$. Since $u \in \overline{\operatorname{co}}\left\{T_{t} x: t \geq s_{0}\right\}$, we have

$$
2\langle z-u, u-z\rangle \geq-\|z-u\|^{2}-\varepsilon
$$

This inequality implies $\|z-u\|^{2} \leq \varepsilon$. Since $\varepsilon$ is arbitrary, we have $z=u$. Therefore

$$
\bigcap_{s \in S} \overline{\operatorname{co}}\left\{T_{t} x: t \geq s\right\} \cap F(\mathscr{S})=\{z\} .
$$

Set $P x=\lim _{t} Q T_{t} x$ for every $x \in C$. Then we have $T_{s} P x=P x$ and

$$
P T_{s} x=\lim _{t} Q T_{t} T_{s} x=\lim _{t} Q T_{t s} x=P x
$$

for every $s \in S$ and $x \in C$. From $\{P x\}=\bigcap_{s \in S} \overline{\operatorname{co}}\left\{T_{t} x: t \geq s\right\} \cap F(\mathscr{S})$, it is obvious that $P x \in \overline{\operatorname{co}}\left\{T_{s} x: s \in S\right\}$ for each $x \in C$. Since

$$
\begin{aligned}
\|P x-P y\| & =\lim _{t}\left\|Q T_{t} x-Q T_{t} y\right\| \leq \limsup _{t}\left\|T_{t} x-T_{t} y\right\| \\
& \leq\left(\limsup _{t} k_{t}\right)\|x-y\| \leq\|x-y\|
\end{aligned}
$$

for every $x, y \in C$, it follows that $P$ is nonexpansive.

We now turn to consider the weak convergence of $\left\{T_{s} x: s \in S\right\}$ and obtain the results similar to these of Lau [7] and Passty [9]. We denote by $\omega(x)$ the set of all weak limit points of subnets of the net $\left\{T_{s} x: s \in S\right\}$. Note that if $F(\mathscr{S})$ is nonempty then $\left\{T_{s} x: s \in S\right\}$ is bounded and hence $\omega(x)$ is nonempty. We start with proving the following lemma. 
LEMMA 4. Let $C$ be a closed convex subset of a Hilbert space $H$ and let $S$ be a right reversible semitopological semigroup. Let $\mathscr{S}=\left\{T_{t}: t \in S\right\}$ be a Lipschitzian semigroup on $C$ with $\lim \sup _{s} k_{s} \leq 1$. Suppose that $F(\mathscr{S}) \neq \varnothing$. Let $x \in C$. If $\omega(x) \subseteq F(\mathscr{S})$, then the net $\left\{T_{s} x: s \in S\right\}$ converges weakly to some $y \in F(\mathscr{S})$.

ProOF. By Lemma 3, the net $\left\{Q T_{s} x: s \in S\right\}$ converges strongly to some $y \in$ $F(\mathscr{S})$. Since $\omega(x) \subseteq \bigcap_{s \in S} \overline{\operatorname{co}}\left\{T_{t} x: t \geq s\right\}$, we have

$$
\bigcap_{s \in S} \overline{\operatorname{co}}\left\{T_{t} x: t \geq s\right\} \cap F(\mathscr{S}) \supseteq \omega(x) \neq \varnothing .
$$

Hence, as in the proof of Theorem 2, we obtain

$$
\{y\}=\bigcap_{s \in S} \overline{\operatorname{co}}\left\{T_{t} x: t \geq s\right\} \cap F(\mathscr{S})
$$

Therefore we have $\{y\}=\omega(x)$.

Let $C$ be a closed convex subset of a Hilbert space $H$ and let $\mathscr{S}=\left\{T_{t}: t \in S\right\}$ be a Lipschitzian semigroup on $C$. A subset $G$ of $S$ is called a uniformly generating set of $\mathscr{S}$ if for each $s \in S$ and $\varepsilon>0$, there exist $g_{1}, g_{2}, \ldots, g_{m} \in G$ such that

$$
\left\|T_{s} x-T_{g_{1} g_{2} \cdots g_{m}} x\right\|<\varepsilon
$$

for every $x \in C$.

THEOREM 3. Let $C$ be a closed convex subset of a Hilbert space $H$ and let $S$ be a right reversible semitopological semigroup. Let $\mathscr{S}=\left\{T_{t}: t \in S\right\}$ be a Lipschitzian semigroup on $C$ with $\lim \sup _{s} k_{s} \leq 1$. Suppose that $F(\mathscr{S}) \neq \varnothing$. Let $x \in C$. If $\lim _{s}\left\|T_{g s} x-T_{s} x\right\|=0$ for all $g$ in a uniformly generating set $G$ of $\mathscr{S}$, then the net $\left\{T_{s} x: s \in S\right\}$ converges weakly to some $y \in F(\mathscr{S})$.

PrOOF. By Lemma 4, it suffices to show that $\omega(x) \subseteq F(\mathscr{S})$. Let $\left\{T_{s_{\alpha}} x\right\}$ be a subnet of $\left\{T_{s} x: s \in S\right\}$ converging weakly to some $y \in C$. Let $\varepsilon>0$. Then for each $t \in S$, there exist $g_{1}, g_{2}, \ldots, g_{m} \in G$ such that

$$
\left\|T_{t} T_{s_{\alpha}} x-T_{g_{1} g_{2} \cdots g_{m}} T_{s_{\alpha}} x\right\|<\varepsilon
$$

for every $\alpha$. Hence we have

$$
\begin{aligned}
\left\|T_{s_{\alpha}} x-T_{t} y\right\| \leq & \left\|T_{s_{\alpha}} x-T_{g_{1} g_{2} \cdots g_{m} s_{\alpha}} x\right\|+\left\|T_{g_{1} g_{2} \cdots g_{m} s_{\alpha}} x-T_{t s_{\alpha}} x\right\|+\left\|T_{t s_{\alpha}} x-T_{t} y\right\| \\
\leq & \left\|T_{s_{\alpha}} x-T_{g_{1} g_{2} \cdots g_{m} s_{\alpha}} x\right\|+\varepsilon+k_{t}\left\|T_{s_{\alpha}} x-y\right\| \\
\leq & \left\|T_{s_{\alpha}} x-T_{g_{1} s_{\alpha}} x\right\|+\left\|T_{g_{1} s_{\alpha}} x-T_{g_{1} g_{2} \cdots g_{m} s_{\alpha}} x\right\|+\varepsilon+k_{t}\left\|T_{s_{\alpha}} x-y\right\| \\
\leq & \left\|T_{s_{\alpha}} x-T_{g_{1} s_{\alpha}} x\right\|+k_{g_{1}}\left\|T_{s_{\alpha}} x-T_{g_{2} \cdots g_{m} s_{\alpha}} x\right\|+\varepsilon+k_{t}\left\|T_{s_{\alpha}} x-y\right\| \\
\leq & \left\|T_{s_{\alpha}} x-T_{g_{1} s_{\alpha}} x\right\|+k_{g_{1}}\left\|T_{s_{\alpha}} x-T_{g_{2} s_{\alpha}} x\right\|+k_{g_{1}} k_{g_{2}}\left\|T_{s_{\alpha}} x-T_{g_{3} s_{\alpha}} x\right\| \\
& +\cdots+k_{g_{1}} k_{g_{2}} \cdots k_{g_{m-1}}\left\|T_{s_{\alpha}} x-T_{g_{m} s_{\alpha}} x\right\|+\varepsilon+k_{t}\left\|T_{s_{\alpha}} x-y\right\|
\end{aligned}
$$

and

$$
\underset{\alpha}{\limsup }\left\|T_{s_{\alpha}} x-T_{t} y\right\| \leq \varepsilon+k_{t} \limsup _{\alpha}\left\|T_{s_{\alpha}} x-y\right\| .
$$

Since $\varepsilon$ is arbitrary, we have

$$
\underset{\alpha}{\limsup }\left\|T_{s_{\alpha}} x-T_{t} y\right\| \leq k_{t} \limsup \left\|T_{s_{\alpha}} x-y\right\| .
$$


On the other hand, since $y$ is the asymptotic center of $\left\{T_{s_{\alpha}} x\right\}$ in $C$ we obtain

$$
\limsup _{t} r\left(T_{t} y\right) \leq\left(\limsup _{t} k_{t}\right) r(y) \leq r
$$

and hence $T_{t} y \rightarrow y$. Therefore we have $y \in F(\mathscr{S})$.

REMARK. Let $\gamma$ be a positive real number and let $\mathscr{S}=\left\{T_{t}: t \in S\right\}$ be a Lipschitzian semigroup with $\limsup _{s} k_{s} \leq \gamma$. Then, putting $k_{s}^{\prime}=\sup _{t \geq s} k_{t}$, we have

$$
\left\|T_{s} x-T_{s} y\right\| \leq k_{s}\|x-y\| \leq \sup _{t \geq s} k_{t}\|x-y\|=k_{s}^{\prime}\|x-y\|
$$

and $\lim _{s} k_{s}^{\prime}=\lim \sup _{s} k_{s}$. Hence $\mathscr{S}$ is a Lipschitzian semigroup with $\lim _{s} k_{s}^{\prime} \leq \gamma$.

\section{REFERENCES}

1. J. B. Baillon, Un théorème de type ergodique pour les contractions non linéaires dans un espace de Hilbert, C. R. Acad. Sci. Paris. Sér. A-B 280 (1975), 1511-1514.

2. K. Goebel and S. Reich, Uniform convexity, hyperbolic geometry, and nonexpansive mappings, Marcel Dekker, New York, 1984.

3. K. Goebel, W. A. Kirk and R. L. Thele, Uniformly lipschitzian families of transformations in Banach space, Canad. J. Math. 26 (1974), 1245-1256.

4. N. Hirano and W. Takahashi, Nonlinear ergodic theorems for nonexpansive mappings in Hilbert space, Kodai Math. J. 2 (1979), 11-25.

5. H. Ishihara and W. Takahashi, Fixed point theorems for uniformly lipschitzian semigroups in Hilbert spaces, J. Math. Anal. Appl. 127 (1987), 206-210.

6. H. Ishihara, Fixed point theorems for lipschitzian semigroups (to appear).

7. A. T. Lau, Semigroup of nonexpansive mappings on a Hilbert space, J. Math. Anal. Appl. 105 (1985), 514-522.

8. T. C. Lim, On asymptotic centers and fixed points of nonexpansive mappings, Canad. J. Math. 32 (1980), 421-430.

9. G. B. Passty, Construction of fixed points for asymptotically nonexpansive mappings, Proc. Amer. Math. Soc. 84 (1982), 212-216.

10. R. R. Phelps, Convex sets and nearest points, Proc. Amer. Math. Soc. 8 (1957), 790-797.

11. W. Takahashi, A nonlinear ergodic theorem for an amenable semigroup of nonexpansive mappings in a Hilbert space, Proc. Amer. Math. Soc. 81 (1981), 253-256.

12. __ A nonlinear ergodic theorem for a reversible semigroup of nonexpansive mappings in a Hilbert space, Proc. Amer. Math. Soc. 97 (1986), 55-58.

Department of Information SCience, Tokyo institute of Technology, OHOKAYAMA, MEgURO-KU, TOKYO 152, JAPAN 